Article

\title{
Efficacy of Lactobacillus Administration in School-Age Children with Asthma: A Randomized, Placebo-Controlled Trial
}

\author{
Chian-Feng Huang ${ }^{1,2}$, Wei-Chu Chie ${ }^{1}$ and I-Jen Wang ${ }^{3,4,5, *(\mathbb{D})}$ \\ 1 Institute of Epidemiology and Preventive Medicine, College of Public Health, National Taiwan University, \\ Taipei 10055, Taiwan; davidbee416@gmail.com (C.-F.H.); weichu@ntu.edu.tw (W.-C.C.) \\ 2 Taoyuan Psychiatric Center, Ministry of Health and Welfare, Taoyuan 33058, Taiwan \\ 3 Department of Pediatrics, Taipei Hospital, Ministry of Health and Welfare, No. 127, Su-Yuan Road, \\ Hsin-Chuang Dist., Taipei 242, Taiwan \\ 4 College of Public Health, China Medical University, Taichung 40402, Taiwan \\ 5 School of Medicine, National Yang-Ming University, Taipei 112, Taiwan \\ * Correspondence: wij636@gmail.com; Tel.: +886-2-2276-5566 (ext.2532); Fax: +886-2-2998-8028
}

Received: 29 September 2018; Accepted: 29 October 2018; Published: 5 November 2018

\begin{abstract}
Probiotics may have immunomodulatory effects. However, these effects in asthma remain unclear and warrant clinical trials. Here, we evaluated the effects of Lactobacillus paracasei (LP), Lactobacillus fermentum (LF), and their combination (LP + LF) on the clinical severity, immune biomarkers, and quality of life in children with asthma. This double-blind, prospective, randomized, placebo-controlled trial included 160 children with asthma aged 6-18 years (trial number: NCT01635738), randomized to receive LP, LF, LP + LF, or a placebo for 3 months. Their Global Initiative for Asthma-based asthma severity, Childhood Asthma Control Test (C-ACT) scores, Pediatric Asthma Severity Scores, Pediatric Asthma Quality of Life Questionnaire scores, peak expiratory flow rates (PEFRs), medication use, the levels of immune biomarkers (immunoglobulin $\mathrm{E}$ (IgE), interferon $\gamma$, interleukin 4 , and tumor necrosis factor $\alpha$ ) at different visits, and the associated changes were evaluated. Compared with the placebo group by generalized estimating equation model, children receiving LP, LF, and LP + LF had lower asthma severity $(p=0.024,0.038$, and 0.007 , respectively) but higher C-ACT scores $(p=0.005,<0.001$, and $<0.001$, respectively). The LP $+\mathrm{LF}$ group demonstrated increased PEFR $(p<0.01)$ and decreased IgE levels $(p<0.05)$. LP, LF, or their combination $(\mathrm{LP}+\mathrm{LF})$ can aid clinical improvement in children with asthma.
\end{abstract}

Keywords: Lactobacillus; probiotics; asthma; Childhood Asthma Control Test; peak expiratory flow rate; immunoglobulin $\mathrm{E}$

\section{Introduction}

Asthma, a chronic complex disease of the airways, is characterized by reversible airflow obstruction, bronchial hyperresponsiveness, and underlying inflammation [1]. The prevalence of asthma has increased in the past decades. A potential mechanism underlying this high prevalence is the microbial hypothesis [2], which argues that less microbial exposure upregulates the cytokine production of T-helper cell type 2 (Th2), leading to an increase in allergic diseases. According to this hypothesis, probiotic administration is an alternative treatment for atopic disease, which when administered in adequate amounts, can confer a health benefit to the host [3]. The researchers found that probiotics have some health effects in atopic disease patients through immunity balancing of T-helper cell type 1 (Th1) and Th2, particularly in those with atopic dermatitis (AD). However, relevant studies focusing on asthma patients are limited. 
A meta-analysis found that, although perinatal and early-life probiotic administration reduces atopic sensitization risk and total immunoglobulin $\mathrm{E}(\mathrm{IgE})$ levels in children, it may not reduce their asthma risk [4]. However, some studies have reported the benefit of using probiotics, in addition to standard care, for treating children with asthma. A randomized, placebo-controlled trial for 7-week Enterococcus faecalis treatment demonstrated decreased peak flow variability in children with asthma [5]. Lee et al. also reported significant improvements in the pulmonary function of children with asthma after a regimen of vegetable, fish oil, and fruit supplementation along with probiotic administration [6]. However, these aforementioned studies were designed as mixed interventions with relatively small sample sizes.

In the present study, we thus included participants representing a population of school-age children with asthma randomized to receive pure strains of Lactobacillus paracasei GMNL-133 (BCRC 910520, CCTCC M2011331) (LP), Lactobacillus fermentum GM-090 (BCRC 910259, CCTCC M204055) (LF), or their mixture (LP + LF). We focused on the therapeutic effects of the probiotics on the disease severity, quality of life, immune biomarkers, and fecal microbial composition in school-age children with asthma.

\section{Materials and Methods}

\subsection{Participants}

This double-blind, randomized, placebo-controlled trial was conducted between December 2011 and September 2013 at the pediatric outpatient clinics of Taipei Hospital, Ministry of Health and Welfare. The inclusion criteria were 6-18 years of age with a history of intermittent to moderate persistent asthma (Global Initiative for Asthma (GINA) steps 1-3) for at least 1 year. We excluded children who had received immunosuppressants, antibiotics, systemic corticosteroids, or antimycotics within 4 weeks before study enrolment or antihistamines within 3 days before study enrolment. The children who had an immunodeficiency disease, other major medical problems, or used probiotic preparations within 4 weeks before study enrolment were also excluded. We acquired written informed consent from all the parents in compliance with the principles of the Helsinki Declaration. The Taipei hospital's Institutional Review Board ratified the study protocol (TH-IRB-10-14). The study was registered under trial number NCT01635738.

\subsection{Protocol}

An investigator enrolled the children and sequentially assigned them a patient number associated with a code. Capsules were prepared and coded by GenMont Biotech Inc. (in their Current Good Manufacturing Practice-certified facilities, Tainan, Taiwan) and dispensed by a study nurse. The children were randomized using computer-generated 4-block design lists created by a statistician, with stratification according to age, sex, severity, and current medication use. We assessed the eligibility of 160 recruited children and randomly allocated them to four groups, with 40 participants in each group (Figure 1). The groups were then randomized to receive LP, LF, LP + LF, or placebo for 3 months. All the investigators, study nurses, and participants were blinded to treatment assignment over the study duration. A capsule count was performed monthly to ensure that the capsules were taken as applicable. Randomization code was deciphered only at the end of the trial.

\subsection{Outcome Measures}

The primary outcome was the changes in asthma severity and Childhood Asthma Control Test (C-ACT) scores over 3 months of the intervention compared with baseline. At baseline and follow-up visits at $0,1,2,3$, and 4 months of intervention, we determined GINA-based asthma severity and recorded C-ACT scores, Pediatric Asthma Quality of Life Questionnaire (PAQLQ) scores, Pediatric Asthma Severity Scores (PASSs), peak expiratory flow rates (PEFRs), and medication use. Skin prick test and blood serum analysis were performed at 0 and 3 months of intervention. In addition, fecal 
microbial analysis was performed for comprehensive evaluation before and after the 3-month treatment course. The changes in PAQLQ score, PASS, PEFR, skin prick test reactivity, serum immune biomarker levels, and fecal probiotic microbial composition were the secondary outcomes (Figure 2).

\subsection{Laboratory Methods}

Skin prick tests using commercial extracts were performed to detect asthma-causing allergens, including mite, cockroach, animal dander, egg, milk, and crab allergens [7]. The levels of IgE and other serum immune biomarkers, such as interferon (IFN) $\gamma$, interleukin (IL) 4 , and tumor necrosis factor (TNF) $\alpha$, were measured through enzyme-linked immunosorbent assay [8]. Specific intestinal bacterial strains in the feces were quantified using conventional culture techniques [9].

\subsection{Sample Size Estimation}

Using nQuery Advisor + nTerim 3.0 (Statistical Solutions Ltd., Cork, Ireland), we calculated the number of participants required to detect the presence of any significant differences in C-ACT scores. According to previous data [10], to detect significant differences in the effects of probiotics on C-ACT scores with $90 \%$ power and a 5\% significance level, each study group must include at least 22 participants. To allow for a $20 \%$ loss from ineligibility or withdrawal, we decided to enroll 30 children in each group. After power assessment, we estimated that 120 total participants would suffice and thus scheduled recruitment of 160 children.

\subsection{Statistical Analysis}

The baseline demographic data of the four groups were compared using analysis of variance. Intragroup comparisons for severity, C-ACT scores, PASSs, PAQLQ scores, and immune biomarker levels at baseline and 3 months of treatment were performed using the paired $t$ test. Differences in outcome variables between the treatment and placebo groups over the five visits were evaluated using a generalized estimating equation (GEE) model after adjustment for potential confounders. All children who completed the study were included in the analysis, regardless of their compliance. All reasons for dropouts or premature withdrawal from the study as well as missing values were recorded. Significance was set at a two-tailed $\alpha$ at 0.05 , and all analyses were performed on SPSS (version 21).

\section{Result}

\subsection{Baseline Characteristics of Participants}

Of 160 recruited children, 152 were finally enrolled and randomly assigned to receive LP, LF, LP + $\mathrm{LF}$, or placebo. Figure 1 shows the relevant patient consort diagram, and Table 1 presents the baseline demographic characteristics of 147 children who completed the entire evaluation. The randomization process ensured adequate comparability between the treatment and placebo groups. No statistically significant differences were observed for any demographic, clinical, or functional variables. Moreover, the clinical manifestation of asthma was similar between the two groups. No significant differences were observed in the severity, total serum IgE level, the rate of sensitization to various allergens, the PEFR, or other parameters between the groups at baseline (Table 2). 


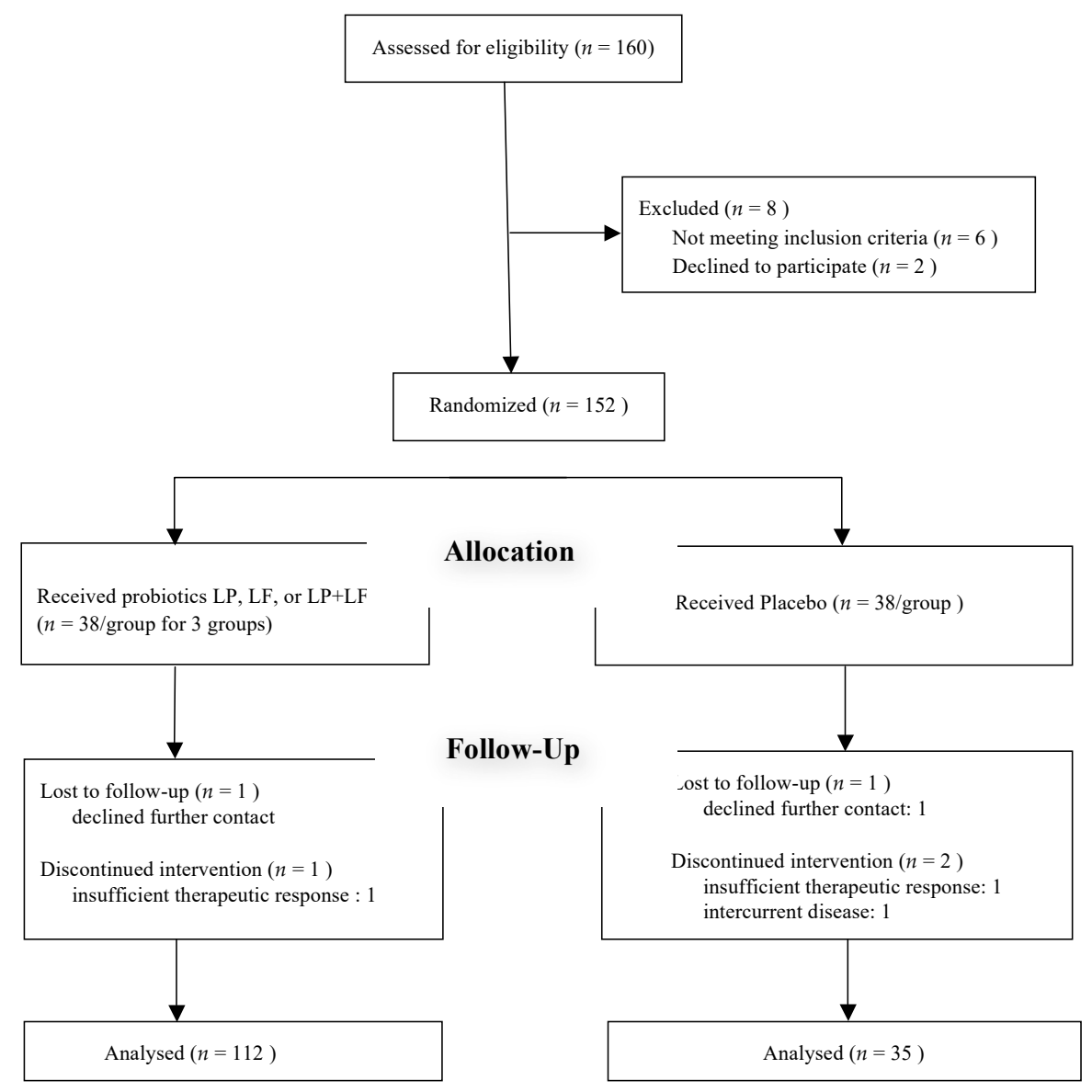

Figure 1. Consort diagram.

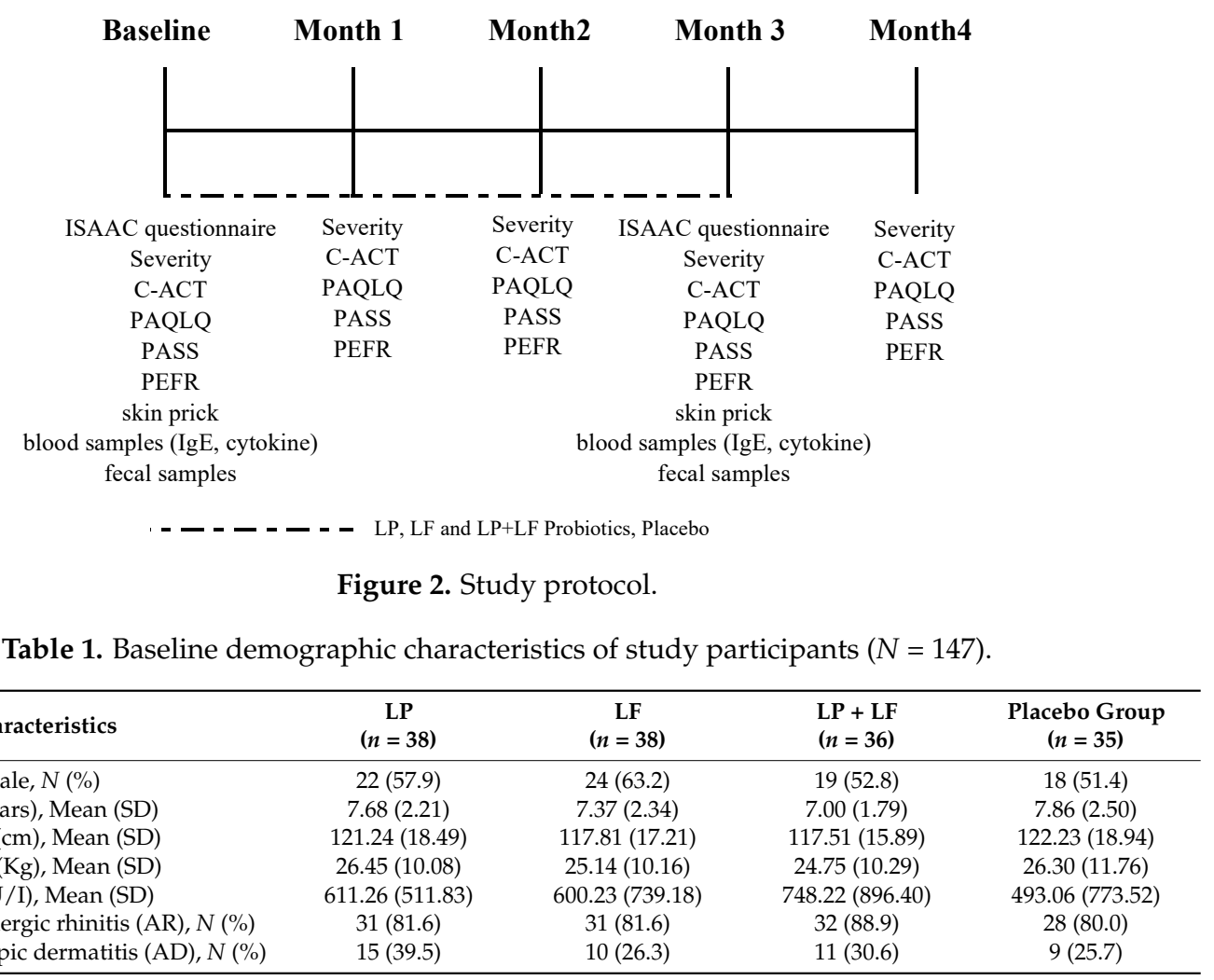


Table 1. Cont.

\begin{tabular}{|c|c|c|c|c|}
\hline Characteristics & $\begin{array}{c}\text { LP } \\
(n=38)\end{array}$ & $\begin{array}{c}\text { LF } \\
(n=38)\end{array}$ & $\begin{array}{l}L P+L F \\
(n=36)\end{array}$ & $\begin{array}{l}\text { Placebo Group } \\
\quad(n=35)\end{array}$ \\
\hline \multicolumn{5}{|l|}{ Allergic sensitization, $N(\%)$} \\
\hline Cockroach & $3(7.9)$ & $2(5.3)$ & $1(2.8)$ & $0(0)$ \\
\hline Animal dander & $2(5.3)$ & $2(5.3)$ & $1(2.8)$ & $1(2.9)$ \\
\hline Milk & $2(5.3)$ & $3(7.9)$ & $1(2.8)$ & $0(0)$ \\
\hline Maternal history of atopic disease, $N(\%)$ & $21(55.3)$ & $22(57.9)$ & $12(33.3)$ & $15(42.9)$ \\
\hline Paternal history of atopic disease, $N(\%)$ & $21(55.3)$ & $21(55.3)$ & $20(55.6)$ & $17(48.6)$ \\
\hline
\end{tabular}

LP, Lactobacillus paracasei; LF, Lactobacillus fermentum.

Table 2. Subscale measures at baseline $(N=147)$.

\begin{tabular}{cccccc}
\hline Subscale & $\begin{array}{c}\text { LP }(n=38) \\
\text { Mean (SD) }\end{array}$ & $\begin{array}{c}\text { LF }(n=38) \\
\text { Mean (SD) }\end{array}$ & $\begin{array}{c}\text { LP + LF }(n=36) \\
\text { Mean (SD) }\end{array}$ & $\begin{array}{c}\text { Placebo }(n=35) \\
\text { Mean (SD) }\end{array}$ & $\begin{array}{c}p \text {-Value } \\
\text { 4 Groups }\end{array}$ \\
\hline Severity & $2.16(0.64)$ & $2.13(0.62)$ & $2.28(0.62)$ & $2.20(0.58)$ & 0.757 \\
C-ACT & $19.89(4.28)$ & $17.87(5.54)$ & $19.81(4.72)$ & $20.77(4.75)$ & 0.074 \\
PAQLQ & $5.44(1.17)$ & $5.40(1.41)$ & $5.90(0.85)$ & $5.58(1.16)$ & 0.268 \\
PASS & $16.84(5.27)$ & $16.29(5.79)$ & $15.71(4.85)$ & $15.60(5.16)$ & 0.738 \\
IFN- $\gamma(\mathrm{ng} / \mathrm{uL})$ & $170.73(188.44)$ & $150.75(158.12)$ & $156.97(167.18)$ & $145.28(162.26)$ & 0.954 \\
IL-4 (ng/uL) & $36.05(29.63)$ & $48.48(85.06)$ & $61.10(117.67)$ & $55.38(86.94)$ & 0.773 \\
TNF- $\alpha(\mathrm{ng} / \mathrm{uL})$ & $292.95(425.14)$ & $324.71(512.82)$ & $231.88(187.97)$ & $207.51(340.37)$ & 0.690 \\
IgE (kU/I) & $611.26(511.83)$ & $600.23(739.18)$ & $748.22(896.40)$ & $493.06(773.52)$ & 0.547 \\
Fecal cell count & & & & & \\
Log10 (CFU/g) & & & & & \\
Lactobacillus & $8.07(0.87)$ & $7.90(0.95)$ & $7.49(1.15)$ & $7.71(0.92)$ & 0.101 \\
Bifidobacterium & $8.81(0.91)$ & $8.74(0.85)$ & $8.75(1.12)$ & $8.90(0.72)$ & 0.872 \\
Clostridium & $7.19(0.78)$ & $6.63(1.09)$ & $6.84(1.18)$ & $6.79(1.11)$ & 0.133 \\
\hline
\end{tabular}

LP, Lactobacillus paracasei; LF, Lactobacillus fermentum; C-ACT, Childhood Asthma Control Test; PAQLQ, Pediatric Asthma Quality of Life Questionnaire; PASS, Pediatric Asthma Severity Scores.

\subsection{Effects of Probiotics on Severity of Asthma and Quality of Life}

Significant intragroup differences were detected in C-ACT scores, the primary outcome, in all groups, except the placebo group (Figure 3). Compared with the placebo group, both asthma severity and C-ACT scores significantly improved in the LP, LF, and LP + LF groups according to our age- and sex-adjusted GEE model (Table 3). No significant difference was noted on both asthma severity and C-ACT scores between the LP + LF and the LP and LF groups, respectively. The PAQLQ scores and PASSs demonstrated no significant group-by-time effects. In the LP + LF group, PEFRs improved significantly $(p<0.01)$.

Table 3. The $p$ values and effect sizes $(\beta)$ compared with the placebo group $(n=35)$ using a generalized estimating equation model.

\begin{tabular}{|c|c|c|c|c|c|c|}
\hline Measure & \multicolumn{2}{|c|}{ LP Group $(n=38)$} & \multicolumn{2}{|c|}{ LF Group $(n=38)$} & \multicolumn{2}{|c|}{ LP + LF Group $(n=36)$} \\
\hline Severity & $-0.34(-0.63,-0.04)$ & $0.024 *$ & $-0.30(-0.59,-0.02)$ & 0.038 * & $-0.43(-0.74,-0.12)$ & $0.007^{*}$ \\
\hline PAQLQ & $-0.32(-0.86,0.23)$ & 0.256 & $-0.30(-0.89,0.28)$ & 0.310 & $-0.43(-0.96,0.09)$ & 0.104 \\
\hline PASS & $-1.39(-4.09,1.30)$ & 0.311 & $-1.14(-3.79,1.51)$ & 0.400 & $-0.48(-3.17,2.20)$ & 0.725 \\
\hline PEFR & $8.77(-11.74,29.27)$ & 0.402 & $28.83(-3.55,61.21)$ & 0.081 & $33.81(8.62,59.00)$ & $0.009 *$ \\
\hline
\end{tabular}

Adjusted for age and sex, ${ }^{*} p<0.05$. LP, Lactobacillus paracasei; LF, Lactobacillus fermentum; C-ACT, Childhood Asthma Control Test; PAQLQ, Pediatric Asthma Quality of Life Questionnaire; PASS, Pediatric Asthma Severity Scores; PEFR, peak expiratory flow rate. 


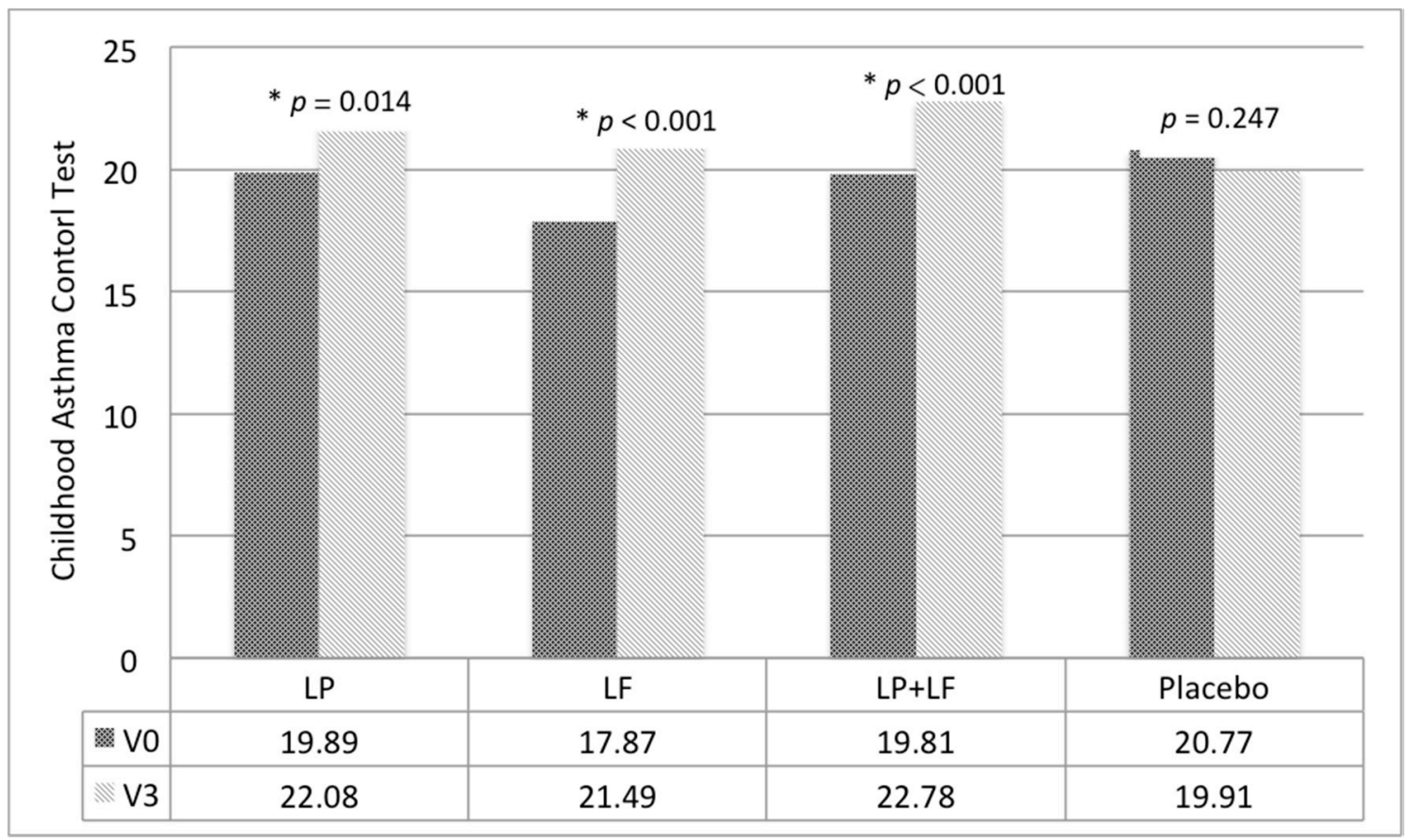

Figure 3. Intragroup differences in Childhood Asthma Control Test scores. ${ }^{*} p<0.05$.

\subsection{Effects of Probiotics on Sensitization and Immune Biomarker Levels}

At the end of treatment, the total serum IgE levels significantly decreased only in the LP + LF group ( $p<0.05$; Table 4$)$. Nevertheless, among all groups, significant intragroup differences, but no intergroup differences, were noted in the skin prick test reactivity to mite allergens. However, no such significant differences were noted for other allergens between before and after the intervention (Table S1). Moreover, serum IFN- $\gamma$, IL-4, and TNF- $\alpha$ levels did not demonstrate any significant changes (Table 4).

Table 4. Immune biomarker levels at baseline and subsequent changes $(N=147)$.

\begin{tabular}{ccccccc}
\hline Subscale & Examination & $\begin{array}{c}\text { LP }(n=38) \\
\text { Mean (SD) }\end{array}$ & $\begin{array}{c}\text { LF }(n=38) \\
\text { Mean (SD) }\end{array}$ & $\begin{array}{c}\text { LP + LF }(n=36) \\
\text { Mean (SD) }\end{array}$ & $\begin{array}{c}\text { Placebo Group }(n=35) \\
\text { Mean (SD) }\end{array}$ & $\begin{array}{c}p \text {-Value } \\
\text { 4 Groups }\end{array}$ \\
\hline IgE & Baseline & $611.26(511.83)$ & $600.23(739.18)$ & $748.22(896.40)$ & $493.06(773.52)$ & 0.547 \\
$(\mathrm{kU} / \mathrm{I})$ & month 3 & $482.42(371.68)$ & $496.40(622.51)$ & $377.29(268.51)^{*}$ & $577.81(705.94)$ & 0.448 \\
IFN- $\gamma$ & Baseline & $170.73(188.44)$ & $150.75(158.12)$ & $156.97(167.18)$ & $145.28(162.26)$ & 0.954 \\
$(\mathrm{ng} / \mathrm{uL})$ & month 3 & $158.01(164.61)$ & $182.75(373.50)$ & $221.41(426.21)$ & $166.21(197.61)$ & 0.886 \\
IL-4 & Baseline & $36.05(29.63)$ & $48.4(85.06)$ & $61.10(117.67)$ & $55.38(86.94)$ & 0.773 \\
$(\mathrm{ng} / \mathrm{uL})$ & month 3 & $51.16(85.33)$ & $49.58(67.82)$ & $55.09(109.40)$ & $104.13(215.39)$ & 0.366 \\
TNF- $\alpha$ & Baseline & $292.95(425.14)$ & $324.71(512.82)$ & $231.88(187.97)$ & $207.51(340.37)$ & 0.690 \\
$(\mathrm{ng} / \mathrm{uL})$ & month 3 & $315.23(625.85)$ & $777.92(1731.71)$ & $584.30(959.98)$ & $550.48(1059.83)$ & 0.514 \\
\hline
\end{tabular}

LP, Lactobacillus paracasei; LF, Lactobacillus fermentum. * $p<0.05$ (Intragroup comparisons).

\subsection{Fecal Microbial Composition, Rescue Medication Use and Compliance}

All intervention groups showed lower counts of Clostridium than did the placebo group. However, these intergroup differences were nonsignificant (Table S2). The frequencies of oral steroid (Table S3), bronchodilator (Table S4), and antihistamine use (Table S5) demonstrated no significant intergroup differences. The compliance of each group is more than $93 \%$ without intergroup differences (Table S6).

\section{Discussion}

The present study effectively resolves the debate regarding whether pure probiotics are beneficial to children with asthma. Using a GEE model, we found that LP, LF, and LP + LF interventions along 
with standard asthma therapy effectively reduced asthma severity and increased C-ACT scores. Furthermore, LP + LF increased both serum IgE levels and PEFRs, implying the existence of a dose-dependent effect.

Consistent with the present study results, a randomized clinical trial by Chen et al. [10] revealed the beneficial effects of probiotics on the severity of asthma and clinical symptoms, but they included participants with asthma accompanied by persistent allergic rhinitis. By contrast, studies have demonstrated the significant effects of various probiotics on some parameters of asthma, but not on its clinical severity, in children with asthma [11,12]. In addition to probiotics containing a single bacterial strain, the clinical advantage of applying a mixture of bacterial strains [13] or using mixed therapy that includes a probiotic regimen [6] has been demonstrated in asthma patients. The reasons for inconsistencies in the aforementioned results may be the variabilities in genetic backgrounds, disease severity, culture, and diet of the participants; moreover, the differences in the study designs, mixed regimens, and strain doses and combinations may also affect the results because the effects of probiotics are strain-specific [14].

We selected LP for this trial because it previously demonstrated beneficial effects in airway hypersensitivity patients [15]. Moreover, it can inhibit related Th2 cytokine production and rebalance the Th1/Th2 immune response by increasing IFN- $\gamma$ levels, as noted in murine bronchoalveolar lavage samples [16]. We also used LF, which can increase IFN- $\gamma$ levels in patients with AD [17] and ameliorate oxidative damage, food allergies, and food-derived infections [18,19]. The present study is the first one in a clinical setting that compared the effects of both single- and mixed-strain probiotics.

Regarding the effects on serum immune biomarker levels, a clinical trial applied single-strain Lactobacillus gasseri A5 probiotics to school children with asthma and allergic rhinitis and reported a nonsignificant reduction in total IgE levels [10]. In the current study, the administration of LF + LP was associated with a significant decrease in IgE levels, while the administration of LP or LF alone showed a tendency to decrease IgE levels, albeit without reaching statistical significance. Similarly, the significant decrease was noted in PEFRs in the LP + LF group. These results may be explained by the synergistic interactions or dose-dependent effects, which were also noted in other probiotic-related trials $[20,21]$; however, the mechanisms underlying these effects remain unclear.

Studies reporting intragroup differences in the effects of probiotics on IgE levels in children with asthma are limited [22]. However, the significance of probiotic-elicited reduction in IgE levels in children with AD has been reported by several studies [23]. Notably, studies have also revealed that, in addition to regulating IgE levels, probiotics can regulate cytokine levels in patients with AD. For instance, in some AD studies [24,25], probiotics significantly reduced serum IL-4 levels, which were compatible with less Th2 responses. However, such cytokine-related findings in the context of asthma are required. The few studies mentioning the related measurement lack consistency in results [10,22].

Notably, based on the preceding comparison of different allergic diseases, gastrointestinal probiotic administration is more efficient at alleviating AD than at alleviating respiratory tract allergic diseases, namely, asthma and allergic rhinitis. Furthermore, the sensitization pattern for asthma allergens, as observed in the current study using the skin prick test, was also different from that for AD allergens. Consistent with previous studies, mites are the predominant aeroallergen of asthma [26-28]. We noted significant postintervention changes in mite sensitization among all groups; however, no intergroup differences were observed. Due to the difference between AD and respiratory tract allergic diseases, it might be useful to consider an alternative method for probiotic administration in children with asthma, such as the intranasal route, which demonstrated effective results in a murine model [29]; however, related human studies are warranted.

Considering the pathogenesis, asthma might be viewed as atopic processes later in life, implying a more systemic level. A review article summarized current experimental data and gave the interpretation of the allergy march; epidermal barrier dysfunction in early life was found to initiate systemic sensitization, which later facilitates the further development of asthma and allergic rhinitis [30]. Another longitudinal study suggested that children with early atopic AD have higher 
risk of asthma than do those with nonatopic AD [31]. Therefore, the administration of a high probiotic dose, probiotic strain mixture, or mixed therapy including probiotics appears suitable for children with asthma compared to other atopic disease; this inference is partly supported by previous trials [6,13].

Regarding fecal intestinal microbiota, the numbers of Lactobacillus, Bifidobacterium, and Clostridium before and after intervention demonstrated no intergroup differences among all four groups. The LP + LF group demonstrated a tendency to elevate the fecal colony counts of Lactobacillus and Bifidobacterium compared to the placebo group, but without reaching statistical significance. It may be explained by the dose-dependent effect: only the combination group received adequate probiotics against consumption. In addition to Lactobacillus, we analyzed the numbers of Bifidobacterium and Clostridium because asthma is associated with a higher amount of Clostridium [32] and a lower level of Bifidobacterium [33] in previous studies, respectively. We found the numbers of Clostridium in LP group tended to decrease after intervention, but without reaching statistical significance. This was probably because Lactobacillus reestablished gut microbiota, as suggested by Durack et al. [34]. The authors asserted that Lactobacillus supplements may recompose gut microbiota, thus preventing atopy and asthma. Furthermore, decreases in Clostridium counts may facilitate colonization by other microorganisms, thus increasing the gut microbial diversity, potentially another protective factor against asthma [35].

This study has some limitations. First, the children with asthma continued receiving standard therapy along with the probiotics, impeding the potential delineation of the effects of probiotics alone. However, the interference is ethical and unavoidable. Moreover, no group differences were noted in oral steroid or bronchodilator use between before and after the intervention. Additionally, these treatments may mask the therapeutic effects of probiotics, which make the results toward the null and strengthen our positive finding. Second, the compliance of the children was another concern. To ensure compliance, we used the capsule counting method rather than the patient record method; this method was easier and less expensive than detection of the study probiotic strains in the feces. Finally, some confounders, including selection bias, host factors (e.g., genetic backgrounds and original microbiota), and environmental factors (e.g., diet and lifestyle) existed. To mitigate this limitation, randomization was performed.

Nevertheless, our study has several strengths: relatively large sample size, comprehensive outcome measures (including C-ACT scores, PAQLQ scores, PASSs, and skin prick testing), and longitudinal repeated measures. We collected different types of data, specifically various serum biomarker levels and fecal microbial compositions. Moreover, a novelty of our study was that we combined two probiotic strains (LP + LF) and noted superior effects in lowering IgE and elevating PEFR than single stain alone. This topic can provide pediatricians, immunologists, and other health care professionals evidence of Lactobacillus administration for childhood asthma. Furthermore, it can also inspire public health experts.

\section{Conclusions}

Our study supports that Lactobacillus is beneficial to children with asthma. We found that both LP and LF can reduce asthma severity and improve asthma control in school-age children. The combination of LP plus LF appears to be more effective in childhood asthma than either LP or LF alone. LP, LF, and their combination were well tolerated with fair compliance and without adverse effects reported.

Supplementary Materials: The following are available online at http:/ / www.mdpi.com/2072-6643/10/11/1678/ s1, Table S1: Skin prick test reactivity, Table S2: Fecal bacterial colony counts, Table S3: Oral steroid use frequency at baseline and during follow-up visits, Table S4: Oral bronchodilator use frequency at baseline and during follow-up visits, Table S5: Oral anti-histamine use frequency at baseline examination and on follow-up visits, Table S6: Probiotics capsules mean taken days monthly record.

Author Contributions: C.-F.H. analyzed and interpreted the data, and wrote the manuscript. W.-C.C. interpreted the data and revised the manuscript. I.-J.W. collected, analyzed, and interpreted the data. I.-J.W. designed the research and revised the manuscript. All authors read and approved the final manuscript. 
Funding: This study was supported by funding from the GenMont Biotec Inc. and by the grants from the National Science Council (NSC 97-2314-B-192-001-MY2) in Taiwan.

Acknowledgments: We thank Miss Ya-Hui Chen and Mr Yi-Hsing Chen for help in the laboratory analysis and related working staff for clinical examination and data collection.

Conflicts of Interest: The Taipei Hospital, the Ministry of Health and Welfare Authority, has a contract with GenMont Biotec Inc. whereby GenMont Biotec Inc. provided the study costs for probiotics. The authors declare no conflict of interest.

\section{References}

1. Shifren, A.; Witt, C.; Christie, C.; Castro, M. Mechanisms of remodeling in asthmatic airways. J. Allergy (Cairo) 2012, 2012. [CrossRef] [PubMed]

2. Kim, H.J.; Kim, H.Y.; Lee, S.Y.; Seo, J.H.; Lee, E.; Hong, S.J. Clinical efficacy and mechanism of probiotics in allergic diseases. Korean J. Pediatr. 2013, 56, 369-376. [CrossRef] [PubMed]

3. Schlundt, J. Report of a Joint FAO/WHO Expert Consultation on Evaluation of Health and Nutritional Properties of Probiotics in Food Including Powder Milk with Live Lactic Acid Bacteria; Food and Agriculture Organization of the United Nations: Córdoba, Argentina, 2001.

4. Ta, V.; Laubach, S. Probiotic administration in early life, atopy, and asthma: A Meta-analysis of clinical trials. Pediatrics 2013, 132, e666-e676. [CrossRef] [PubMed]

5. Stockert, K.; Schneider, B.; Porenta, G.; Rath, R.; Nissel, H.; Eichler, I. Laser acupuncture and probiotics in school age children with asthma: A randomized, placebo-controlled pilot study of therapy guided by principles of Traditional Chinese Medicine. Pedriatr. Allergy Immunol. 2007, 18, 160-166. [CrossRef] [PubMed]

6. Lee, S.C.; Yang, Y.H.; Chuang, S.Y.; Huang, S.Y.; Pan, W.H. Reduced medication use and improved pulmonary function with supplements containing vegetable and fruit concentrate, fish oil and probiotics in asthmatic school children: A randomised controlled trial. Br. J. Nutr. 2013, 110, 145-155. [CrossRef] [PubMed]

7. Duotip-Test ${ }^{\circledR} I I$ Package Insert. Available online: https://www.penallergytest.com/wp-content/uploads/ Duo-Tip-2-Package-Insert.pdf (accessed on 16 September 2018).

8. Shyur, S.-D.; Jan, R.-L.; Webster, J.R.; Chang, P.; Lu, Y.J.; Wang, J.Y. Determination of multiple allergen-specific IgE by microfluidic immunoassay cartridge in clinical settings. Pediatr. Allergy Immunol. 2010, 21, 623-633. [CrossRef] [PubMed]

9. Tsai, C.C.; Chiu, T.H.; Ho, C.Y.; Lin, P.P.; Wu, T.Y. Effects of anti-hypertension and intestinal micro-flora of spontaneously hypertensive rats fed gammaaminobutyric acid-enriched Chingshey purple sweet potato fermented milk by lactic acid bacteria. Afr. J. Microbiol. Res. 2013, 7, 932-940.

10. Chen, Y.S.; Jan, R.L.; Lin, Y.L.; Chen, H.H.; Wang, J.Y. Randomized placebo-controlled trial of lactobacillus on asthmatic children with allergic rhinitis. Pediatr. Pulmonol. 2010, 45, 1111-1120. [CrossRef] [PubMed]

11. Miraglia Del Giudice, M.; Maiello, N.; Decimo, F.; Fusco, N.; D'Agostino, B.; Sullo, N.; Capasso, M.; Salpietro, V.; Gitto, E.; Ciprandi, G.; et al. Airways allergic inflammation and L. reuterii treatment in asthmatic children. J. Biol. Regul. Homeost. Agents 2012, 26, S35-S40. [PubMed]

12. Giovannini, M.; Agostoni, C.; Riva, E.; Salvini, F.; Ruscitto, A.; Zuccotti, G.V.; Radaelli, G. A randomized prospective double blind controlled trial on effects of long-term consumption of fermented milk containing lactobacillus casei in pre-school children with allergic asthma and/or rhinitis. Pediatr. Res. 2007, 62, 215-220. [CrossRef] [PubMed]

13. Miraglia Del Giudice, M.; Indolfi, C.; Capasso, M.; Maiello, N.; Decimo, F.; Ciprandi, G. Bifidobacterium mixture (B longum BB536, B infantis M-63, B breve M-16V) treatment in children with seasonal allergic rhinitis and intermittent asthma. Ital. J. Pediatr. 2017, 43. [CrossRef] [PubMed]

14. Wickens, K.; Black, P.N.; Stanley, T.V.; Mitchell, E.; Fitzharris, P.; Tannock, G.W.; Purdie, G.; Crane, J. A differential effect of 2 probiotics in the prevention of eczema and atopy: A double-blind, randomized, placebo-controlled trial. J. Allergy Clin. Immunol. 2008, 122, 788-794. [CrossRef] [PubMed]

15. Fujiwara, D.; Wakabayashi, H.; Watanabe, H. A double blind trial of Lactobacillus paracasei strain KW3110 administration for immunomodulation in patients with pollen allergy. Allergol. Int. 2005, 54, 143-149. [CrossRef] 
16. Wang, X.; Hui, Y.; Zhao, L.; Hao, Y.; Guo, H.; Ren, F. Oral administration of lactobacillus paracasei 19 attenuates PM2.5-induced enhancement of airway hyperresponsiveness and allergic airway response in murine model of asthma. PLoS ONE 2017, 12. [CrossRef] [PubMed]

17. Prescott, S.L.; Dunstan, J.A.; Hale, J.; Breckler, L.; Lehmann, H.; Weston, S. Clinical effects of probiotics are associated with increased interferon-gamma responses in very young children with atopic dermatitis. Clin. Exp. Allergy 2005, 35, 1557-1564. [CrossRef] [PubMed]

18. El-Ghaish, S.; Rabesona, H.; Choiset, Y.; Sitohy, M.; Haertle, T.; Chobert, J.M. Proteolysis by Lactobacillus fermentum IFO3956 isolated from Egyptian milk products decreases immuno-reactivity of $\alpha \mathrm{S} 1$-casein. J. Dairy Res. 2011, 78, 203-210. [CrossRef] [PubMed]

19. Mikelsaar, M.; Zilmer, M. Lactobacillus fermentum ME-3 an antimicrobial and antioxidative probiotic. Microb. Ecol. Health Dis. 2009, 21, 1-27. [CrossRef] [PubMed]

20. Chang, Y.S.; Trivedi, M.K.; Jha, A.; Lin, Y.F.; Dimaano, L.; Garcia-Romero, M.T. Synbiotics for prevention and treatment of atopic dermatitis: a meta-analysis of randomized clinical trials. JAMA Pediatr. 2016, 170, 236-242. [CrossRef] [PubMed]

21. Szulińska, M.; Łoniewski, I.; van Hemert, S.; Sobieska, M.; Bogdański, P. Dose-dependent effects of multispecies probiotic supplementation on the lipopolysaccharide (LPS) level and cardiometabolic profile in obese postmenopausal women: A 12-week randomized clinical trial. Nutrients 2018, 10, 773. [CrossRef] [PubMed]

22. Lin, J.; Zhang, Y.; He, C.; Dai, J. Probiotics supplementation in children with asthma: A systematic review and meta-analysis. J. Paediatr. Child Health 2018, 54, 953-961. [CrossRef] [PubMed]

23. Kim, S.O.; Ah, Y.M.; Yu, Y.M.; Choi, K.H.; Shin, W.G.; Lee, J.Y. Effects of probiotics for the treatment of atopic dermatitis: A meta-analysis of randomized controlled trials. Ann. Allergy Asthma Immunol. 2014, 113, 217-226. [CrossRef] [PubMed]

24. Wang, I.J.; Wang, J.Y. Children with atopic dermatitis show clinical improvement after Lactobacillus exposure. Clin. Exp. Allergy 2015, 45, 779-787. [CrossRef] [PubMed]

25. Prakoeswa, C.R.; Herwanto, N.; Prameswari, R.; Astari, L.; Sawitri, S.; Hidayati, A.N.; Indramaya, D.M.; Kusumowidagdo, E.R.; Surono, I.S. Lactobacillus plantarum IS-10506 supplementation reduced SCORAD in children with atopic dermatitis. Benef. Microbes 2017, 8, 833-840. [CrossRef] [PubMed]

26. Oncham, S.; Udomsubpayakul, U.; Laisuan, W. Skin prick test reactivity to aeroallergens in adult allergy clinic in Thailand: A 12-year retrospective study. Asia Pac. Allergy 2018, 8. [CrossRef] [PubMed]

27. Arshad, S.H.; Tariq, S.M.; Matthews, S.; Hakim, E. Sensitization to common allergens and its association with allergic disorders at age 4 years: A whole population birth cohort study. Pediatrics 2001, 108. [CrossRef]

28. Vidal, C.; Lojo, S.; Juangorena, M.; Gonzalez-Quintela, A. Association between asthma and sensitization to allergens of Dermatophagoides pteronyssinus. J. Investig. Allergol. Clin. Immunol. 2016, 26, 304-309. [CrossRef] [PubMed]

29. Spacova, I.; Petrova, M.; Fremau, A.; Pollaris, L.; Vanoirbeek, J.; Ceuppens, J.L.; Seys, S.; Lebeer, S. Intranasal administration of probiotic Lactobacillus rhamnosus GG prevents birch pollen-induced allergic asthma in a murine model. Allergy 2018. [CrossRef] [PubMed]

30. Spergel, J.M. From atopic dermatitis to asthma: The atopic march. Ann. Allergy Asthma Immunol. 2010, 105, 99-106. [CrossRef] [PubMed]

31. Novembre, E.; Cianferoni, A.; Lombardi, E.; Bernardini, R.; Pucci, N.; Vierucci, A. Natural history of "intrinsic" atopic dermatitis. Allergy 2001, 56, 452-453. [CrossRef] [PubMed]

32. Stiemsma, L.T.; Arrieta, M.C.; Dimitriu, P.A.; Cheng, J.; Thorson, L.; Lefebvre, D.L.; Azad, M.B.; Subbarao, P.; Mandhane, P.; Becker, A.; et al. Shifts in Lachnospira and Clostridium sp. in the 3-month stool microbiome are associated with preschool age asthma. Clin. Sci. 2016, 130, 2199-2207. [CrossRef] [PubMed]

33. Hevia, A.; Milani, C.; López, P.; Donado, C.D.; Cuervo, A.; González, S.; Suarez, A.; Turroni, F.; Gueimonde, M.; Ventura, M.; et al. Allergic patients with long-term asthma display low levels of Bifidobacterium adolescentis. PLoS ONE 2016, 11. [CrossRef] [PubMed] 
34. Durack, J.; Kimes, N.E.; Lin, D.L.; Rauch, M.; McKean, M.; McCauley, K.; Panzer, A.R.; Mar, J.S.; Cabana, M.D.; Lynch, S.V. Delayed gut microbiota development in high-risk for asthma infants is temporarily modifiable by Lactobacillus supplementation. Nat. Commun. 2018, 9. [CrossRef] [PubMed]

35. Abrahamsson, T.R.; Jakobsson, H.E.; Andersson, A.F.; Bjorksten, B.; Engstrand, L.; Jenmalm, M.C. Low gut microbiota diversity in early infancy precedes asthma at school age. Clin. Exp. Allergy 2014, 44, 842-850. [CrossRef] [PubMed]

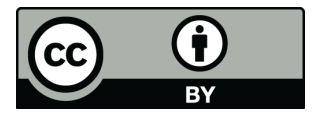

(C) 2018 by the authors. Licensee MDPI, Basel, Switzerland. This article is an open access article distributed under the terms and conditions of the Creative Commons Attribution (CC BY) license (http://creativecommons.org/licenses/by/4.0/). 